\title{
Real-time simulation of surgical cutting in haptic environments using computational vademecums
}

\author{
Carlos Quesada ${ }^{1}$, Icíar Alfaro ${ }^{1}$, David González ${ }^{1}$, Elías Cueto ${ }^{1}$, Francisco Chinesta ${ }^{2}$ \\ ${ }^{1}$ Applied Mechanics and Bioengineering (AMB) \\ Instituto de Investigación en Ingeniería de Aragón (I3A) \\ Universidad de Zaragoza, Mariano Esquillor s/n, 50018, Zaragoza, Spain. \\ Tel. +34-976762707, e-mail: cquesada@unizar.es \\ ${ }^{2}$ Ecole Centrale de Nantes
}

\begin{abstract}
A method for the real-time simulation of surgical cutting in haptic environments is presented. It relies on the use of computational vademecums, i.e. PGDbased, pre-computed meta-models, which allow the solution to be obtained at fast rates. An example for corneal surgery is provided.
\end{abstract}

\section{Introduction and objective}

The interest in training surgeons using computer simulators is well-known, although it entails significant difficulties. In particular, a convincing, realistic, real-time simulation of the surgical cut in soft tissue is a major source of difficulties. Firstly, making incisions involves modifying the geometry and the topology of the organ and its associated mesh. And secondly, real-time simulators impose restrictive response speeds $[1,2]$.

To overcome the real-time constraint, different approaches have been developed during the past decades. Model order reduction (MOR) techniques allows to develope models with a reduced amount of components that run properly under strict time limitations [3]. As for the geometry, extended finite element methods (X-FEM) have been successfully implemented with other MOR techniques [4].

The work proposed here introduces a method to simulate real-time surgical cut using haptic (tactile) devices, which require a highly demanding response time $(\sim 1 \mathrm{kHz})$ to provide the user a realistic touch sensation. The method uses the MOR methodology known as Proper Generalized Decomposition (PGD). For the first time, it has been successfully coupled with X-FEM to simulate the cutting procedure. PGD is presented in the form of a computational vademecum [5], i.e. a meta-model, computed beforehand for each possible value of all the parameters under consideration, which can be evaluated quickly at runtime.

\section{Technical aspects}

A summary of the methodology employed to achieve the objective is presented below.

\section{PGD in a nutshell}

Proper Generalized Decomposition (PGD) is a model order reduction methodology based on the use of separated representations (or finite sum decompositions). PGD allows the solution $u(\boldsymbol{x})$ of a $d$-parametrical boundary value problem to be approximated as

$u\left(x_{1}, x_{2}, \ldots, x_{d}\right) \approx \sum_{i=1}^{N} F_{1}^{i}\left(x_{1}\right) \cdot F_{2}^{i}\left(x_{2}\right) \cdot \ldots \cdot F_{d}^{i}\left(x_{d}\right)$,

where both the number of terms $N$ and the functions $F_{j}^{i}\left(x_{j}\right)$ are unknown a priori. The price to pay is the non-linear character of the representation; however, the gain is clearly significant since its performance is impressive [6].

The PGD approximation $u(\boldsymbol{x})$ is constructed by successive enrichment, using an iterative scheme whereby each functional product is determined alternately, in sequence, till convergence is achieved. The number of terms $N$ in the PGD separated representation is often a few tens.

\section{Real-time cut simulation}

In this approach, the approximation to the model is obtained as a sum of the displacements of a continuous part (not accounting for the cuts) and a discontinuous part:

$$
u=u^{\text {cont }}+u^{\text {disc }} .
$$

The process by which this solution is obtained can be divided into two stages: an off-line one, previous to the simulation execution; and an on-line one, which takes place at runtime. 


\section{Off-line stage}

In this stage, PGD is used to obtain a different $u^{\text {cont }}$ for each load state, i.e. for each position $\boldsymbol{s}$ of the organ on which a cutting tool (e.g. a scalpel) may apply a force. To achieve this, PGD is computed over the weak form of the linear elasticity equations.

On the other hand, X-FEM techniques are also used to obtain the additional displacements $u^{\text {disc }}$ produced on the organ by the cuts. This is achieved by using discontinuous enrichment functions. The displacements are computed for any possible orientation $\theta$ and position $\boldsymbol{s}$ of the cut. The real path of the cut will be approximated during the on-line stage by applying those displacements to the nearest nodes in the model mesh [7].

Depending on the coarseness of the approximation, the computation of both $u^{\text {cont }}$ and $u^{\text {disc }}$ may be a really time-consuming process. Yet, it is a once-ina-lifetime task.

\section{On-line stage}

The previously computed $u^{\text {cont }}$ and $u^{\text {disc }}$ are used in this stage as a reduced basis to obtain the approximation $u$ :

$$
u(\boldsymbol{x}, \boldsymbol{s}, \theta)=\beta_{i} \cdot u_{i}{ }^{\mathrm{cont}}(\boldsymbol{x}, \boldsymbol{s})+\gamma_{j} \cdot u_{j}{ }^{\mathrm{disc}}(\boldsymbol{x}, \boldsymbol{s}, \theta),
$$

The new variables $\beta_{i}$ and $\gamma_{j}$, which can be seen as a sort of weighting parameters, are the only unknowns in this system of equations. From a computational point of view, the system can be solved as a simple matrix system. The solving process takes place at runtime and fulfills the requirements on response time. As new cuts are applied to the structure, new equations appear in the system, which manifest as new rows and columns in the matrix.

\section{Application to corneal surgery}

An example of corneal surgery simulation (radial keratotomy) has been developed. This kind of surgery consists of making radial incisions in the corneal tissue with a diamond knife to eliminate myopia or astigmatism. A computer cornea model, consisting of 8514 nodes and 6840 elements was used. Hyperelastic material behavior was assumed. The model was subjected to different patterns of radial cuts (Fig. 1 shows one of them).

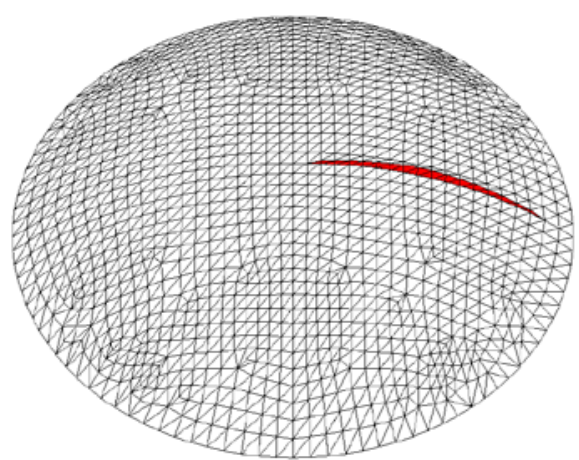

Fig. 1: Radial cut in cornea model.

\section{Conclusions}

Both the appearance and the haptic response produce a very realistic sensation. Response time improves previous approaches. As for the quality of the solution, $\mathrm{L}_{2}$-error norms were computed using a FEM simulation as a reference. In all cases they remained below $5 \cdot 10^{-3}$, which is considerably lower than other similar simulations.

\section{REFERENCES}

[1]. BIELSER, D. and GROSS, M.H. Interactive simulation of surgical cuts. In: The Eighth Pacific Conference on Computer Graphics and Applications, 2000 - Proceedings. Piscataway: IEEE, 2000, pp. 116-442.

[2]. DelingetTE, H. and AYACHE, N. Soft tissue modeling for surgery simulation. In: Computational Models for the Human Body, Handbook of Numerical Analysis. Oxford: Elsevier, 2004, pp. 453-550.

[3]. NIROOMANDI, S., et al. Real-time deformable models of non-linear tissues by model reduction techniques. Computer Methods and Programs in Biomedicine. 2008, 91(3), 223-231.

[4]. NIROOMANDI, S., et al. Model order reduction in hyperelasticity: a proper generalized decomposition approach. International Journal for Numerical Methods in Engineering. 2013, 96(3), 129-149.

[5]. CHINESTA, F. et al. PGD-Based Computational Vademecum for Efficient Design, Optimization and Control. Archives of Computational Methods in Engineering. 2013, 20(1), 31-59.

[6]. CHINESTA, F., KEUNINGS, R. and LEYGUE, A. The Proper Generalized Decomposition for Advanced Numerical Simulations. A primer. New York: Springer, 2014.

[7]. BELYTSCHKO, T. and SONG J.H. Cracking node method for dynamic fracture with finite elements. International Journal for Numerical Methods in Engineering. 2008, 77(3), 360-385. 